\title{
Electrocardiosignals and Motion Signals Telemonitoring and Analysis System for Sportsmen
}

\author{
S Korsakas ${ }^{1}$, A Vainoras ${ }^{1,2}$, V Miskinis ${ }^{1}$, R Ruseckas ${ }^{1}$, V Jurkonis ${ }^{1}$, \\ R Jurkoniene ${ }^{1}$, K Berskiene ${ }^{2}$, L Siupsinskas ${ }^{2}$, V Marozas ${ }^{3}$ \\ ${ }^{1}$ Institute of Cardiology of Kaunas University of Medicine, Lithuania \\ ${ }^{2}$ Departament of Kinesiology and Sports Medicine of Kaunas University of Medicine, Lithuania \\ ${ }^{3}$ Biomedical Engineering Institute of Kaunas University of Technology, Lithuania
}

\begin{abstract}
The aim of this paper is to present a new electrocardiosignals (ECGs) and motion signals telemonitoring and analysis system for sportsmen paddling a canoe or kayak. The developed system is intended to facilitate the couch in optimizing and individualizing the training of elite athletes.

The hardware system consists of rower and coach components. Rower components include 5 sensors for monitoring of mechanical and physiological parameters. The coach components include master unit and laptop computer. The coach software works in two modes: online version during training and off-line the detailed data analysis after training. The new method for systolic blood pressure evaluation was developed and checked for 313 persons. The accuracy of systolic blood pressure prediction could exceed range of $90 \%$.

The preliminary results of developed system testing and method for blood pressure evaluation are promising and show that telemetry system could be used for monitoring of ECG and motion parameters when sportsman is in action.
\end{abstract}

\section{Introduction}

Literature analysis shows active interests of application of high-tech IT technologies in high level athlete sports [1]. The company WEBA has developed KAYAK ERGOMETER which gives recreational and top-athletes the best opportunity to simulate the motion stereotype of paddle sport [2]. There are several goals sought: increasing the performance of sportsmen by logging the results during training, online or offline analysis and presentation of the analysis results to the couch and sportsmen, preventing injuries during rehabilitation of sportsmen (project DROMEAS) [3]. However, the amount of information they provide is not enough for the coach. Usually only one physiological parameter, i. e., the heart rate is measured. The physiological parameters are needed for functional state evaluation of sportsman for optimising his performance during training process [4]. The aim of this study was to develop the telemetry system for sportsmen which satisfied the requirements for training in real conditions and should execute on-line and off-line data analysis.

\section{Methods}

Human organism is a complex dynamic adaptive system. To evaluate its performance, adaptation to physical load, we need to measure developed power and the changes in organism which where evoked by this power. In case of canoe rowing the force and speed sensors helps to record main dynamic parameters of canoe. When we know force function during every row, it's possible to calculate the mean of force in time interval $\Delta \mathrm{T}$ :

$$
\mathrm{F}_{\text {mean }}=1 / \Delta \mathrm{T} * \Sigma \mathrm{F}_{\mathrm{i}}^{*} \Delta \mathrm{t}_{\mathrm{i}}
$$

In the same way could be calculated the mean of speed:

$$
\mathrm{V}_{\text {mean }}=1 / \Delta \mathrm{T} * \Sigma \mathrm{V}_{\mathrm{i}}^{*} \Delta \mathrm{t}_{\mathrm{i}}
$$

The mean power will be the product of calculated means:

$$
\mathrm{N}_{\text {mean }}=\mathrm{F}_{\text {mean }} * \mathrm{~V}_{\text {mean }}
$$

When we know developed mean power, it is important to evaluate the productivity of human organism in every time period. Directly to measure it is very difficult task, usually we have to many influences from outside and it is not possible to take into account all them. So usually are used approximations of parameters which can't be measured directly during training, but could be evaluated during special stress tests. During such stress test could be evaluated connection between needed parameters for individual person. Using human organism functional state model [4], during stress test the connection between heart rate (HR), systolic blood pressure $(\mathrm{S})$ and relative heart power (measured by the changes of JT interval, which reflects the speed of metabolic processes in the heart). It is possible only from ECG to evaluate the changes of $\mathrm{S}$, and to calculate

$$
S=f(H R, J T)
$$


The pressure is directly connected to inner human organism power of its performance, so $N_{\text {in }}=f(S)$ for individual person. After calculations of discussed two powers $\mathrm{N}_{\text {mean }}$ and $\mathrm{N}_{\text {in }}$ could be evaluated the productivity of human organism:

$$
\eta=\mathrm{N}_{\text {mean }} / \mathrm{N}_{\text {in }} \text {. }
$$

This relation can reflect common dynamic characteristics of the complex system 'human organism - canoe'. Also the performance of human organism could be evaluated using the parameters discussed earlier. Summary value of such performance could be calculated as a distance in Euclidian space:

$$
\mathrm{S}_{\mathrm{v}}=\mathrm{k}\left((\Delta \mathrm{RR}) 2+(\Delta \mathrm{JT}) 2+\left(\Delta \mathrm{N}_{\mathrm{in}}\right) 2\right) 1 / 2 \text {. }
$$

The changes of $S_{v}$ in time could allow the evaluation of human organism adaptation to load, the changes of functional state.

Method of the systolic blood pressure evaluation. Earlier developed human functional state model during physical activity [4] for the systolic blood pressure calculation was used. For the evaluation of the systolic blood pressure we studied the double product:

$$
D P=H R \cdot S \cdot 10^{-2} \text {. }
$$

We analyzed the functional relation between double product and JT interval during physical load. This relation we postulated by hyperbolic function $(\mathrm{N}>50 \mathrm{~W})$ and by linear function $(\mathrm{N} \leq 50 \mathrm{~W})$ :

$$
D P= \begin{cases}\frac{a}{J T}-b, & N>50 \mathrm{~W}, \\ -k J T+m, & N \leq 50 \mathrm{~W} .\end{cases}
$$

We calculated parameters $a, b, k$ and $m$ by the least squares method for each person [5].

For the evaluation of systolic blood pressure we used the parameters a,b,k,m from previous test and the new data HR and JT interval observed during next test:

$$
S= \begin{cases}\frac{a-b \cdot J T}{\check{S} S D \cdot J T} \cdot 10^{2}, & N>50 \mathrm{~W}, \\ \frac{-k J T+m}{\check{S} S D} \cdot 10^{2} & N \leq 50 \mathrm{~W} .\end{cases}
$$

For the accuracy of evaluation we compared the calculated systolic blood pressure value with observed value and counted the relative percentage error.

The investigated contingent consisted of asymptomatic 313 persons (107 men and 206 women, they were tested two times). All persons were divided into 6 groups according to gender and age $(20-30,30-40$ and $40-50$ years old). For evaluation of cardiovascular system reactions the ECG analysis system "Kaunas - Load", developed at the Institute of Cardiology, Kaunas University of Medicine was used [6]. There were used the standard stress test method of provocative incremental bicycle ergometry work. The bicycle ergometry work was started from $50 \mathrm{~W}$ power $(\mathrm{N})$, increasing the power every minute by $25 \mathrm{~W}$ for women and $50 \mathrm{~W}$ for men. We used these functional indexes during load: the heart rate (HR), the systolic arterial blood pressure $(\mathrm{S})$, JT interval sum in 12 leads (JT) at rest and in each level of load [6].

\section{Results}

The relation between double product and JT interval during physical load was approximated by hyperbolic and linear function by least square method. The relative percentage error of approximation was less than 5\% ( $\mathrm{p}<0,05$ for all groups. In figure 1 is given the example of real and approximated DP(JT) for 30 - 40 years old women group.

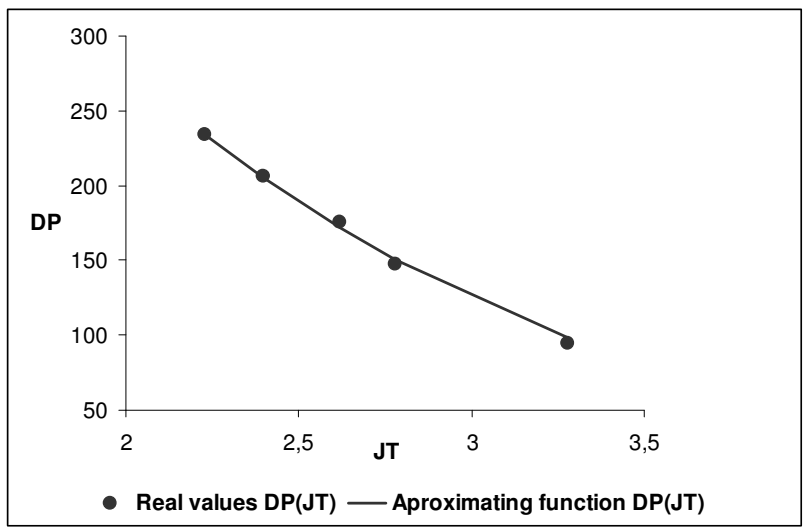

Figure 1. The relation between DP and JT interval for 30 -40 years old women group.

During first test by (1) formula we calculated parameters a, b, k, m. During next test we calculated the systolic blood pressure by (2) formula. We estimated that the accuracy of systolic blood pressure prediction could exceed range of $90 \%$ if the person's functional state is stable and the tests are in short time interval $(1-2$ months). Prediction example is given in figure 2 .

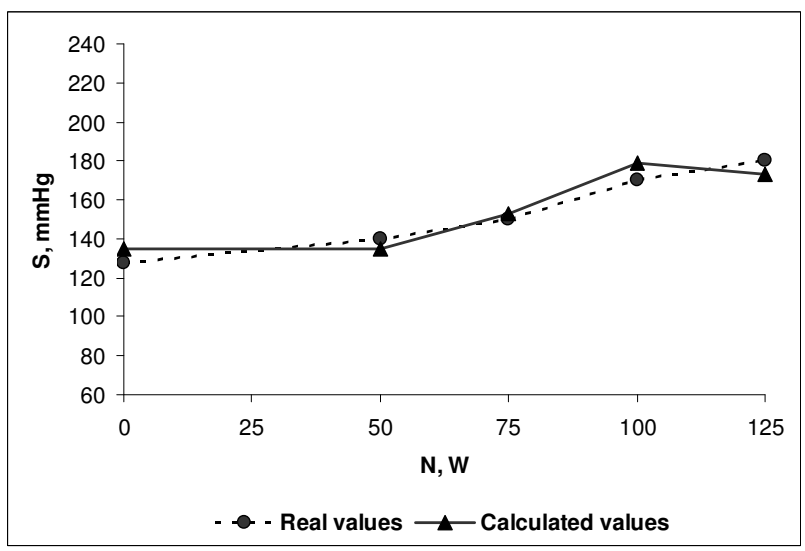

Figure 2. The example of real and calculated systolic blood pressure for one investigator. 
If the tests are in long time interval (6 or more months) and the functional state is not stable (illness, injuries) the relative percentage error of systolic blood pressure evaluation exceeds $20 \%$. We checked, that relative percentage error of systolic blood pressure were less than $15 \% \quad(\mathrm{p}<0,05)$ for all investigators.

The component diagram of the developed telemonitoring system is presented in figure 3 .

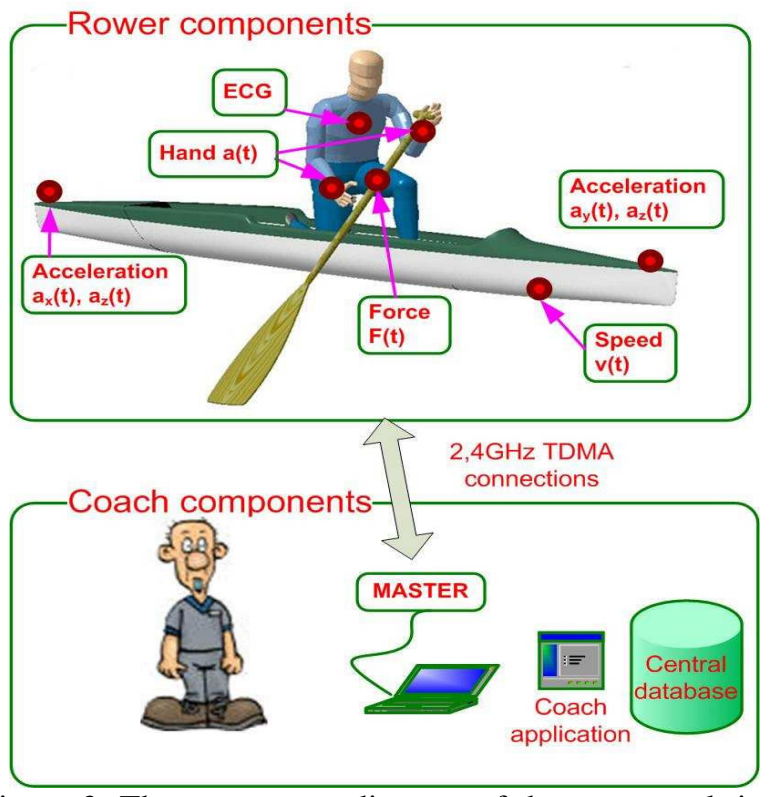

Figure 3. The component diagram of the rower real time telemonitoring system.

It consists of rower and coach components. Rower components include 5 sensors for monitoring of mechanical and physiological parameters. The coach components include master unit, laptop computer, coach application, central database of signals. Master unit and sensors form the $2,4 \mathrm{GHz}$ wireless, proprietary star topology sensor network. The aggregated data from the master flow to the laptop computer. The following physiological and mechanical parameters are monitored: a) three EASI ECG leads, b) two motion signals from arms or legs, c) force to the paddle- $F(t)$, d) speed of the boat $\mathrm{v}(\mathrm{t})$. Additionally acceleration of the stern of the boat in $X$ and $Z$ axes- $a_{x}(t), a_{z}(t)$ and acceleration of the bow of the boat in $\mathrm{Y}$ and $\mathrm{Z}$ axes- $\mathrm{a}_{\mathrm{y}, \mathrm{z}}(\mathrm{t}), \mathrm{a}_{\mathrm{z}}(\mathrm{t})$ parameters are measured using Analog Devices Inc. ADXL202 MEMS type accelerometers. The accelerations of the bow and stern are measured in order to estimate the synchronization of vertical movements of the ends of the boat. In order to go faster it is important to keep up these movements in phase.

The developed software works in two modes: on-line version during training and off-line for the detailed data analysis after training. On-line software consists of next program modules: data input, calculation heart rate (HR) and repolarization (ST slope) parameters, visualization input signals (ECG and Motion) and data recording in the PC memory. Figure 4 presents the screenshot of parameters HR, ST, 4 channels of motion signals and 3 EASI ECG leads in real time.

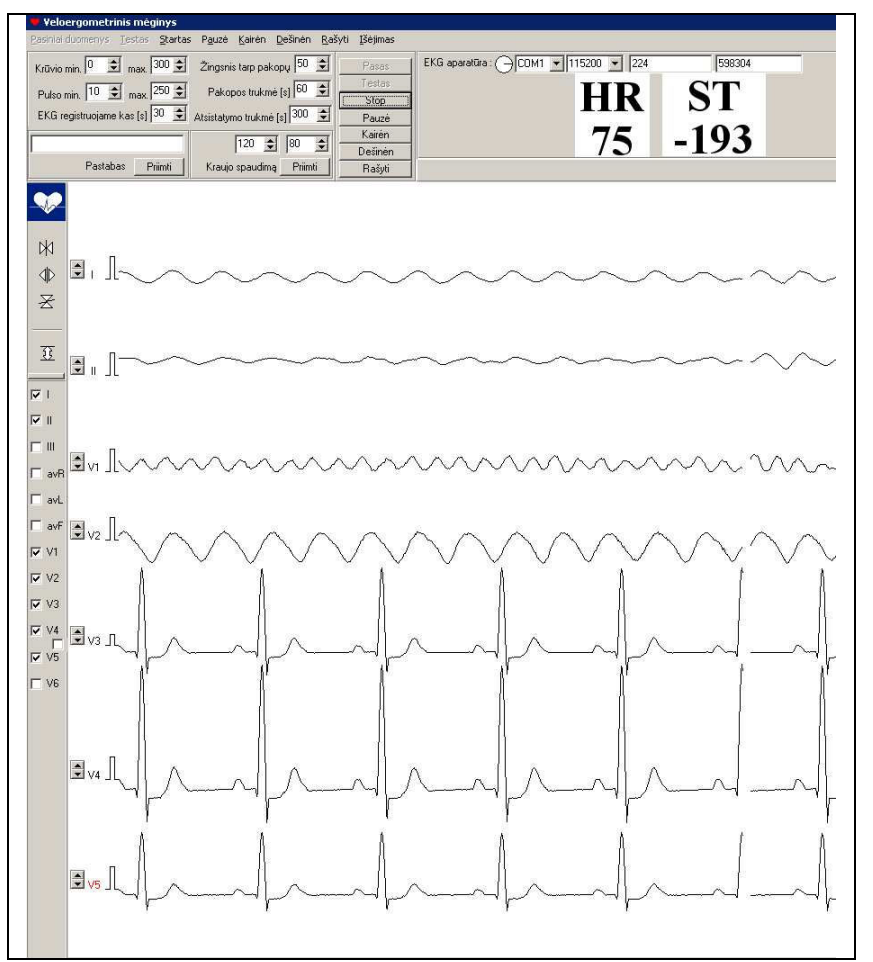

Figure 4. The screenshot of parameters HR, ST, 4 channels of motion signals and 3 EASI ECG leads.

Off-line software consists of program modules: power evaluation from motion signals, transformation from 3 EASI leads to 12 standard ECG leads, systolic blood pressure evaluation and program package "KaunasLoad".

Primary the developed hardware and software of telemonitoring system for ECGs and motion signals recording and analysis was tested in laboratory. Laboratory testing involved synchronously recorded standard 12 lead ECG and EASI system ECG with motion signals during standard exercise test. We have assessed 14 young (average -16 years) cyclists. The aim of research was to compare data from standard 12 lead ECG with data, registered on our ECGs and motion signals telemonitoring system. The software diagram for comparing results of data analysis with both systems is presented in figure 5. Motion signals were taken from two accelerometers, placed on each of the legs of cyclists. 
Each accelerometer registered motion signals of the legs in two axes.

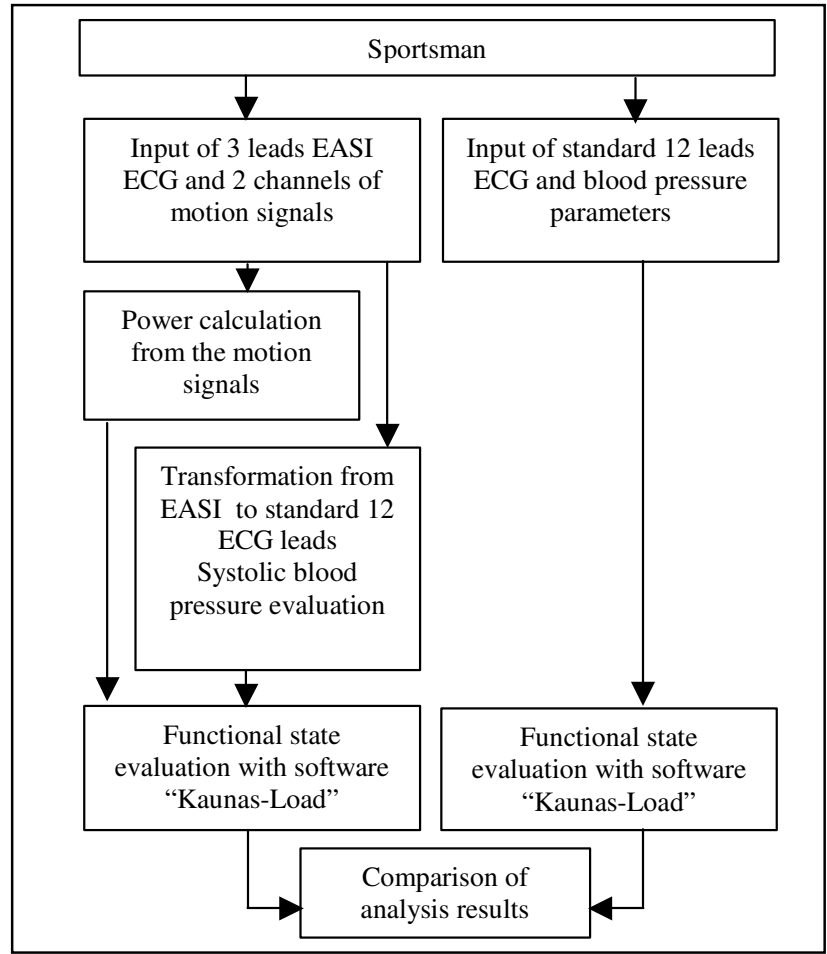

Figure 5. The software diagram for comparison results of data analysis during exercise test of sportsman.

Because the cyclist acceleration was constant during exercise test, the power was not possible to calculate from motion signals from legs of cyclists. This method is good for rowers only. Information from registered EASI system of ECG was converted into standard 12 lead system. The systolic blood pressure was evaluated using our developed method (see above). Analysis of data was done using ECG analysis system "Kaunas-Load". Comparison of analysis results of real 12 ECG leads and converted from 3 EASI into 12 leads (Table 1) shows that parameters $S_{v}$ (integral evaluation functional state of sportsman during physical load), $\mathrm{HR}_{0}, \mathrm{HR}_{\mathrm{m}}, \mathrm{ST}_{0}, \mathrm{ST}_{\mathrm{m}}$ are not differ significantly.

Table 1. Comparison results of real 12 ECG leads and converted from 3 EASI into 12 leads

\begin{tabular}{|l|l|l|l|l|l|}
\hline Param & $\mathrm{S}_{\mathrm{v}}$ & $\mathrm{HR}_{0}$ & $\mathrm{HR}_{\mathrm{m}}$ & $\mathrm{ST}_{0}$ & $\mathrm{ST}_{\mathrm{m}}$ \\
\hline$\Delta \mathrm{M}$ & 5.25 & -13.7 & 11.9 & 0.14 & -0.12 \\
\hline$\delta$ & 9.86 & 23.3 & 21.8 & 0.48 & 0.17 \\
\hline $\mathrm{SEM}$ & 2.85 & 6.73 & 6.3 & 0.14 & 0.05 \\
\hline
\end{tabular}

ECGs and motion signals telemonitoring system was tested in real conditions too. Some tests were done on water on the rower of kayak. We put ECG electrodes on the body, accelerometers on the hands and synchronously recorded 3 EASI ECG leads and two motion signals, transmitting them to the laptop computer. Quality of the signals was satisfied, area of communication approximately 60 meters. The main advantage of this system is ability to record, transmit, visualisation, analyse ECG and transmit alarm signal to couch or sportsman in real time (on-line).

\section{Discussion and conclusions}

The prototype of ECGs and motion signals telemonitoring and analysis system for sportsmen paddling a canoe or kayak is designed and implemented. The main advantage of this system is ability to record, transmit, visualisation, analyse ECG and transmit alarm signal to couch or sportsman in real time (on-line). Another new aspect of the system is assessment of the physical status of the sportsman by analysing the transmitted ECG on-line and comprehensive analysis offline. In conclusion, the preliminary results of testing developed hardware and software are promising and show that ECGs and motion signals telemonitoring and analysis system could be used for rowers.

\section{Acknowledgements}

This work was supported by a grant from the Lithuanian Science and Studies Fund for project named HOMOTECH.

\section{References}

[1] NK rowing computers. Internet site address: http://www.nkhome.com/rp/rpindex.html

[2] WEBA KAYAK Ergometer. Internet site address: http://www.weba-sport.com/kayak_expert.html

[3] EU FP5 IST program project DROMEAS, http://www.dromeas.eu.com/index.html

[4] Vainoras A. Functional model of human organism reaction to load - evaluation of sportsman training effect. Education Physical Training Sport 2002; 3:88-93.

[5] Pekarskas V. The differential and the integral calculation II part. Kaunas; 2000.

[6] Vainoras A., Gargasas L., Ruseckas R., Miskinis V., Jurkoniene R., Shwela H., Bauer U.J. Computerized exercise electrocardiogram analysis system "KaunasLoad"،, In BACHAROVA L. and MACFARLANE P.W. (Ed): 'Electrocardiology'97', World Scientific, SingaporeNew Jersey-London-Hong Kong 1998:253-6

Address for correspondence

Name Stasys Korsakas

Full postal address Sukileliu 17, LT50009 Kaunas, Lithuania

E-mail address stakor@medi.lt 